\title{
Improving Video Streaming Over IEEE 802.11 Mesh Networks through a Cross-Layer Design Technique
}

\author{
N. H. Moleme ${ }^{*}$, M.O. Odhiambo ${ }^{*}$, A.M. Kurien ${ }^{* *}$ \\ French South African Technical Institute in Electronics \\ Tshwane University of Technology, Private Bag X680 Pretoria 0001 Republic of South Africa \\ Tel: (+27 12) 318-4191 Fax: (+27 12) 318-5294 \\ nametshego.m@gmail.com,ohangmo@unisa.ac.za ,kurienam@tut.ac.za
}

\begin{abstract}
There has been a spate of interest in wireless mesh networks in recent years. The reason behind such an upsurge of interest is that wireless mesh networks are seen as enablers of ubiquitous broadband access applications such as the Internet. Moreover, wireless mesh networks provides benefits such as ease of installation, cost effective deployments, a high level of scalability in coverage area and capacity, network flexibility and self-configuration capabilities. These benefits enable the proliferation of seamless communication in underdeveloped areas such as rural communities. However, despite all these benefits, many research challenges still remain in wireless mesh networks. One such challenge is the support of real time applications such as video streaming. This paper presents a cross-layer optimization technique for real time applications provisioned over IEEE 802.11 wireless multihop networks. In this technique, packet error control and throughput optimization is established through rate adaptation mechanisms. Congestion control and optimal route selection in the network layer is achieved through routing protocol optimizations. Simulation results show that the proposed cross-layer optimization method significantly improves the performance of multihop wireless mesh networks when UDP is used as the transport protocol.
\end{abstract}

Index Terms-Cross-Layer, Video Streaming, Rate Adaptation, Routing Protocol.

\section{INTRODUCTION}

$\mathrm{W}$ ireless Mesh Networks (WMNs) have gained immense research interest from the wireless networks research society in recent years. This sudden interest emanates from developments which indicate that WMNs can offer ubiquitous communication and seamless broadband applications. WMNs are hybrid networks considered to be variants of Wireless Ad Hoc Networks (WANETs) as they are built based on a mixture of fixed and mobile nodes interconnected via wireless links to form a multihop WANET [1],[2]. Thus, WMNs inherit most, if not all of the multihop WANETs characteristics. There are numerous possible video streaming applications over multihop wireless mesh networks. Applications such as spontaneous video conferencing in a place without wireless infrastructure, Internet Protocol Television (IPTV), Video on Demand
(VoD), video transmission on the battlefield, search and rescue operations, security and surveillance systems [3]. However, there are still several research challenges that need to be addressed in all protocol layers for WMNs to support video streaming applications. In this study, efforts are focused on transport layer protocols.

Transmission Control Protocol (TCP) and User Datagram Protocol (UDP) are two de facto transport layer protocols used within the wireless network technology. The majority of research studies based on these protocols reveal that TCP is unsuitable for real-time applications [4]. TCP incorporates retransmissions and congestion control mechanisms. These mechanisms results in additional delays and jitter. Video streaming applications can tolerate a certain level of packet losses. However, they are very sensitive to delay and jitter. Moreover, congestion window based transport protocols are typically related to busty data rates. This characteristic makes them unsuitable for video streaming applications which normally prefer steady data rates. For this reason, UDP is the most preferred transport layer protocol for real time applications.

A significant amount of packet loss in wireless networks is due to wireless channel errors and network congestion. UDP does not incorporate error recovery mechanisms like TCP. Thus, video streaming in wireless networks using UDP the protocol can result in unpredictable degradation and poor video quality. In addition, UDP throughput degrades with an increased hop count between the sender and the receiver in wireless multihop networks. This behaviour is expected since packet error probability is maximized when more hops are encountered from source to destination [5]. To improve the performance of IEEE 802.11 multihop wireless mesh networks for video streaming applications, a mechanism that optimizes throughput, minimizes packet loss and improves end-to-end delays for UDP protocol is needed.

In this paper, a cross-layer optimization technique based on the data link and network layers for UDP optimization is proposed. The IEEE $802.11 \mathrm{~b}$ is used as the benchmark standard to evaluate the proposed cross-layer technique. However, with slight modifications, the cross-layer design can be easily adapted to IEEE $802.11 \mathrm{a} / \mathrm{g}$ standards.

The rest of this paper is organized as follows. Section II, gives a description of different automatic rate control approaches used for rate adaptation and the implementation of the approach that is used in this study. Section III, discusses routing protocol optimizations. In Section IV, 
simulation results are shown. Section V concludes the paper and discusses future work.

\section{RATE ADAPTATION}

The cross-layer optimization technique in this study comprises of two components: rate adaptation and routing optimization. In this section, rate adaptation and its construction is described. In the following section, the routing protocol optimization and implementation is described. The illustration of the proposed cross-layer technique is shown in Figure 1.
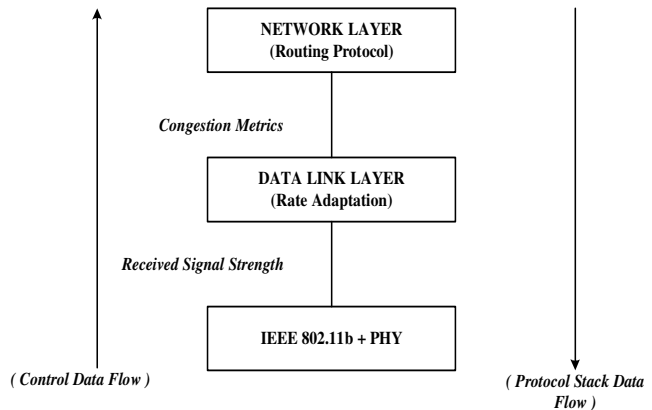

Figure 1: Implementation of the Cross-Layer Design in the Protocol Stack

\section{A. Rate Control Approaches for Rate Adaptation}

There are different types of rate control approaches that exist in wireless networks for rate adaptation implementation. In this section, source based automatic rate control, receiver based automatic rate control, statistics based automatic rate control and Signal-to-Noise (SNR) based automatic rate control which constitute the four main approaches used within the wireless network framework are discussed.

\section{Source Based Automatic Rate Control}

In this approach, the sender single-handedly regulates the transmission rate. This is achieved through monitoring transmission failures (packet losses). This approach is depicted in Automatic Rate Fallback (ARF) scheme described in [18]. The ARF scheme enables the source node to reduce its current transmission rate if two consecutive transmission failures occur. This prompts the node to start running a timer. The current transmission rate is then raised to a higher transmission rate when the timer count expires or 10 packet acknowledgements are successfully received. The timer is then reset. When the transmission rate is increased, the probing transmission (first transmission after the rate increase) must be successful or else the transmission rate is immediately decreased and the timer count is restarted. This means that a higher rate will only be tried once. If it is not successful, a lower rate will be chosen. ARF has two major disadvantages. Firstly, if the frequency of channel conditions variations is very high, the scheme is unable to adapt efficiently. Secondly, if channel conditions remain unchanged or vary slowly, ARF will try to use a higher transmission rate after every 10 successfully transmitted packets. This results in increased retransmission attempts and thus decreases the application throughput
To alleviate the problems presented by ARF, Adaptive Auto Rate Fallback (AARF), which is an extension of ARF, was proposed in [19]. In AARF, when the first transmission after the rate increase fails, the current rate is immediately changed to the previous lower rate similar to ARF. However, the number of consecutive successful transmissions required to switch to a higher rate is multiplied by two. The rationale behind this approach is that throughput can be greatly optimized if packet failures take up a large amount of transmission time.

\section{Receiver Based Automatic Rate Control}

In this approach, the receiver plays a role in assisting the sender to efficiently regulate the transmission rate. This is achieved by using feedback information from the receiver. The most prevalent receiver based automatic rate control scheme is Receiver Based Auto Rate (RBAR). RBAR makes use of the Request To Send (RTS) and Clear To Send (CTS) mechanisms to enable the receiver to calculate the transmission rate for the sender. When the node receives the RTS, it estimates the transmission rate based on the channel conditions indicated by the SNR. The receiver then piggybacks the transmission rate into the CTS packet and sends it back to the source. The source finally uses the transmission rate that has been piggybacked into the CTS packet to transmit its data. In RBAR, the size and rate of the data transmission is inserted in the RTS and CTS packets to enable all the nodes in the surrounding areas to update their Network Allocation Vector (NAV) through overhearing.

Opportunistic Auto Rate (OAR) is proposed in [20] to improve the performance of receiver based rate control in terms of throughput. OAR makes use of the RTS and CTS mechanisms similar to RBAR. However, the principle of OAR is to enable multiple packet transmission at higher rates by allowing a flow to hold the channel until a sufficient number of packets are transmitted when the channel is of good quality. Thus, OAR achieves more throughput as more packets are sent at higher transmission rates when compared to RBAR. The major drawback of these control schemes is that they require incompatible changes to the IEEE 802.11 RTS and CTS mechanisms. Thus, it is doubtful that they will ever be deployed in existing IEEE 802.11 devices.

\section{Statistics Based Automatic Rate Control}

Statistics based rate control schemes use statistical feedback information about channel conditions to enable the transmitter to select an appropriate rate for packet transmission. Statistical information such as Frame Error Rate (FER), number of retries per frame and acknowledged transmissions are used [21]. This is depicted in FER based auto rate scheme. In this scheme, the FER of the transmitted frames is utilized to choose a suitable rate for data stream transmission. In IEEE 802.11, FER is easily acquired as correct reception of data frames by the receiver node is explicitly acknowledged by an acknowledgement message. Thus, an unacknowledged frame is assumed to be lost by the sender. FER is calculated as a function of received frames over transmitted frames within a limited period of time. In FER based auto rate mechanism, two methods are used for rate selection.

- Downscaling: If FER is more than certain set threshold and the present transmission rate is not 
the minimal rate, then the rate is changed over to the next lower rate.

- Upscaling: If FER is near zero, the link is probed at the adjacent higher transmission rate. If acknowledged, the rate is changed over to the transmission rate.

\section{SNR Based Automatic Rate Control}

In this approach, the sender uses the SNR to select an appropriate rate for transmission. The SNR is either acquired as feedback information from the receiver or a channel model is used to estimate the channel conditions which are indicated by the SNR. Link adaptation rate control scheme proposed in [12] is based on this approach. In this scheme, the transmitter selects an appropriate transmission rate based on the Received Signal Strength (RSS) determined from Access Point (AP) frames. The transmitter uses the RSS as the Channel State Indicator (CSI). RSS variations signal changes in the wireless link. Thus, rate adaptation is initiated. Rate control is achieved by using threshold techniques. When the average RSS determined from AP frames exceed certain set thresholds the rate is adapted. All the stations use 12 thresholds. These thresholds are initialized to zero and are dynamically updated when the station is in operation.

\section{B. Implementation of Rate Adaptation}

The two most common and prevalent rate control approaches for rate adaptation is statistic based automatic rate control and SNR based automatic rate control [17]. Statistics based scheme delivers robust performance and optimized throughput in the long run. However, it responds very slowly to channel variations. This can be catastrophic to real time applications. In contrast to statistics based rate control, the SNR-based rate control has a very fast response. However, the problem of the approach is that in practice, SNR is not easily available for devices to use. Secondly, the channel models utilized for channel conditions estimation (SNR) are inadequate. Nevertheless, it is still the most efficient available approach if an efficient channel model is utilized.

The principle of this technique is to dynamically change the data transmission rate based on the SNR which is used as the channel quality estimation parameter. Bit-Error Rates (BER) and Frame Error Rates (FER) are dependent on the SNR in IEEE 802.11 networks as illustrated in Figure 2. The empirical curves shown in Figure 2 are for an Intersil chip known as HFA3861B [12]. These curves indicate the variation of BER as a function of SNR based on the specific modulation schemes, Binary Phase Shift Keying (BPSK), Quadrature Phase Shift Keying (QPSK) and Complimentary Code Keying (CCK). In IEEE 802.11b protocol, BPSK and QPSK modulation schemes are used for $1 \mathrm{Mbps}$ and $2 \mathrm{Mbps}$ transmission rates respectively and CCK is used for both 5.5 and $11 \mathrm{Mbps}$ transmission rates.

Maximal throughput is achieved if an appropriate transmission rate (Modulation scheme) is used for specific channel conditions indicated by SNR as shown in Figure 3. For instance, it is shown that at an SNR of approximately $10 \mathrm{~dB}$, data can be transmitted at both $5.5 \mathrm{Mbps}$ and $11 \mathrm{Mbps}$ transmission rates. The $5.5 \mathrm{Mbps}$ transmission rate yields BER of $10^{-6}$ while the $11 \mathrm{Mbps}$ yields a BER of $10^{-4}$. The receiver might be able to receive packets with both BERs depending on the receiver sensitivity. However, throughput will not be optimized.

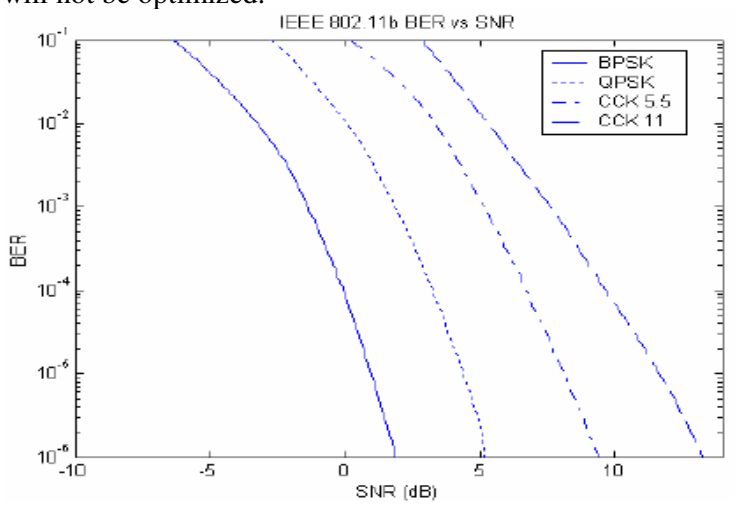

Figure 2: IEEE 802.11b BER vs. SNR [12]

Thus, to optimize throughput, an appropriate selection of the transmission rate (modulation scheme) based on the SNR is required. This can be achieved through the use of an automatic rate controller which is used to dynamically select an appropriate transmission rate for an optimal throughput [17]

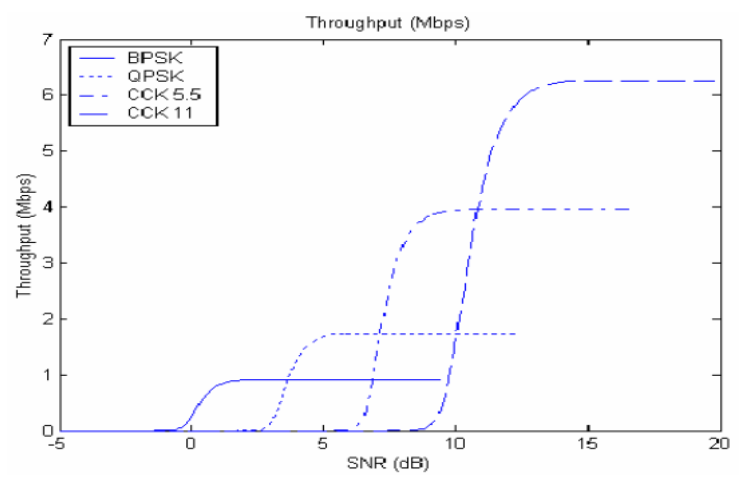

Figure 3: IEEE 802.11b Throughput vs. SNR [17]

The rate adaptation scheme comprises of three components: channel model, throughput model and automatic rate controller. The channel model is utilized for channel quality estimations based on channel conditions. The throughput model estimates the attainable throughput based on the channel quality estimate. The rate controller automatically selects an appropriate transmission rate based on the channel quality estimation and the maximum attainable throughput.

\section{Channel Model}

The channel model selection is based on the practical experimental evaluations of the log-distance model conducted in [9], [10]. Practical measurements were conducted using off-the-shelf IEEE 802.11 hardware equipment to validate the use of the log-distance model in simulations. The experimental results showed that the logdistance model can be used successfully in simulations to yield accurate results. The log-distance channel model is utilized in the rate adaptation scheme. It is expressed as follows. 


$$
\overline{P_{\text {Loss }}(d B)}=\overline{P_{\text {Loss }}\left(D_{0}\right)}+10 \cdot \alpha \cdot \log \left(\frac{D}{D_{0}}\right),
$$

where $D_{0}$ is the close-in reference distance, $D$ is the distance between the source node and the destination node, and $\alpha$ the path loss exponent specified in [11].

\section{Throughput Model}

The throughput model for the rate adaptation is based on [8], [12], [13]. Throughput is a function of packet length and the average time taken to transmit the packet. In IEEE 802.11, a packet is enclosed in a MAC Service Data Unit (MSDU) from the network layer to the MAC layer as shown in Figure 4 which illustrates the transmitted frame format. Thus, the packet length refers to the MSDU length and the average time taken to transmit a packet refers to the time taken to transmit a Physical Layer Convergence Procedure Protocol Data Unit (PPDU).

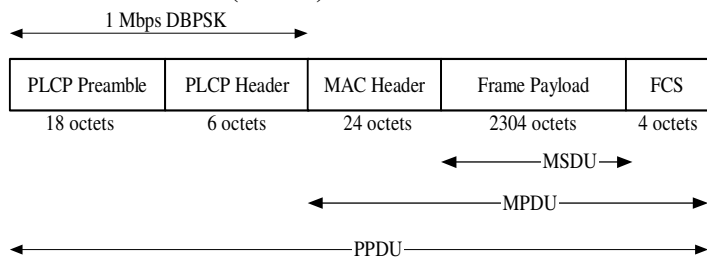

Figure 4: IEEE 802.11 Transmitted Frame [13]

$T_{P P D U_{-} \text {frame }}$ is the average time taken to transmit a frame including retransmissions and is modeled under the MAC protocol Distributed Coordination Function (DCF) mechanism with Request to Send (RTS) and Clear to Send (CTS) disabled [8]. To model $T_{P P D U_{-} \text {frame }}$, the probability of a frame error needs to be known. This is given by the following expression.

$$
P_{P P D U_{-} \text {Error }}=1-\left(1-P_{\text {Overhead_Error }_{1}}\right) \cdot\left(1-P_{M P D U_{-} \text {Error }}\right)
$$

$P_{\text {Overhead_error }}$ is the physical layer overhead error probability and $P_{M P D U_{-} \text {Error }}$ is the MAC Protocol Data Unit (MPDU) error probability. The physical layer overhead error probability is given by the following expression.

$$
P_{\text {Overhead_Error }}=1-\left(1-P_{b_{-} \text {err } 1}\right)^{(18+6) .8},
$$

where $P_{b_{-} \text {err } 1}$ is the bit error probability when transmitting the physical layer overhead. The physical layer overhead is always transmitted on 1Mbps Differential Binary Phase Shift Keying (DBPSK). The MPDU error probability is given by the following expression.

$$
P_{M P D U_{-} \text {Error }}=1-\left(1-P_{b_{-} \text {err } 2}\right)^{(24+4+M S D U) \cdot 8},
$$

where $P_{b_{-} e r r 2}$ is the bit error probability when transmitting the MPDU. $T_{P P D U_{-} \text {frame }}$ is then expressed as follows.

$$
T_{P P D U_{-} \text {frame }}=T_{S}(0)+\sum_{i=1}^{+\infty}\left(1-p_{\text {PPDU_Error }}\right) \cdot\left(P_{P P D U_{-} E \text { Error }}\right)^{i}\left[\sum_{j=0}^{i-1} T_{\varphi}(j)+T_{\psi}(i)\right] \mu s \text {, }
$$

where $T_{\varphi}(i)$ is the time taken to successfully transmit a frame including retransmissions and $T_{\psi}(j)$ is time between two consecutive frame transmissions after a frame transmission failure. Throughput is then expressed as follows.

$$
\operatorname{Throughput}\left(L_{M S D U}, T_{P P D U_{-} \text {frame }}\right)=\left(\frac{L_{M S D U} \cdot 8}{T_{P P D U_{-} \text {frame }}}\right) M b p s,
$$

where $L_{M S D U}$ is the length of the MSDU in bytes

\section{Automatic Rate Controller}

For the automatic rate controller, a simple threshold based technique was used. In this technique, the transmission rate is chosen based on the channel quality estimation and the maximum attainable throughput under the estimated channel quality conditions. The Receive Signal-Strength (RSS) is used as the channel quality estimator since it is a more practical parameter that is available than SNR in the $N S-2$ Simulator. The transmission power of each MSDU is known. Therefore, the receive power (RSS) is estimated using the channel model indicated in equation (1). The RSS is then mapped to a transmission data rate based on the estimated maximum attainable throughput modeled in equation (6). The mapping of the channel conditions to a transmission rate is achieved by obtaining thresholds from the throughput vs. RSS curves. These curves are plotted and discussed in section IV.

\section{ROUTING OPTIMIZATIONS}

To optimize the routing protocol in the network layer for congestion and optimal path selection, the Dynamic Source Routing (DSR) protocol [14] is modified to utilize congestion information from MAC layer and link quality metric in the network layer.

\section{A. Dynamic Source Routing Protocol}

It has been shown through several conducted performance comparisons studies that reactive routing protocols such as DSR generally perform better than proactive routing protocols such as Destination-Sequenced Distance Vector (DSDV). This is attributed to their performance in terms of routing overheads. DSR has been shown to be more efficient in WANETs when compared to other reactive routing protocols such as Ad Hoc On-Demand Distance Vector (AODV) as a result of employing source routing mechanism [22].

The DSR protocol comprises of a Route Discovery and a Route Maintenance mechanisms [23]. The Route Discovery mechanism is used to establish source routes, while the Route Maintenance mechanism is used to maintain the established routes and to detect any changes to these routes. Source routing eradicates the problem of loops which is associated with protocols that periodically broadcasts routing information such as DSDV. It also enables nodes that are sending or overhearing packets to cache the routing information for their own future use. Since DSR is totally an on-demand protocol, its overhead is significantly lower as it only reacts to changes in the routes currently in use.

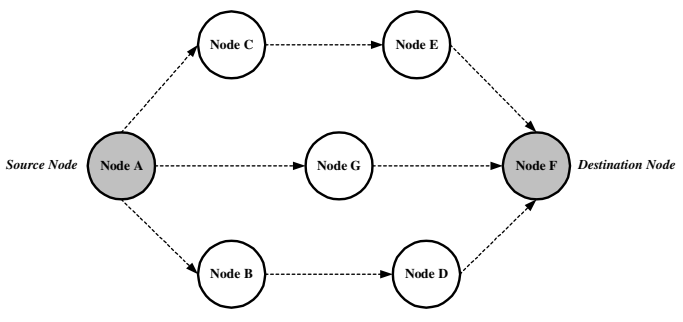

Figure 5: Multihop Network Layout Scenario 
The DSR protocol utilizes the Route Discovery mechanism to establish a communication path between node $\mathrm{A}$ and node $\mathrm{F}$ if the source node $\mathrm{A}$ needs to communicate with the destination node $\mathrm{F}$ and node $\mathrm{A}$ cannot directly communicate with node $\mathrm{F}$ as shown in Figure 5. To execute the Route Discovery process, node A broadcasts a Route Request (RREQ) message containing the destination node's (node F) address and a request identification. The RREQ is disseminated throughout the entire wireless multihop network. If node $G$ receives the RREQ, it executes a verification process. In this process, node $G$ determines if it has recently received an RREQ from node A containing the same request identification and destination address, or alternatively, if its own address has already been registered on the RREQ. If one of the two checks is determined to be true, the RREQ will be discarded. If none is true, node $G$ will register its own address on the RREQ and forward it to destination node F. In this instance, it is assumed that node $\mathrm{G}$ has not yet registered a route to node $\mathrm{F}$ in its route cache. Otherwise, node $\mathrm{G}$ would have returned a Route Reply (RREP) packet to node A. This RREP would have contained a list of nodes forming a communication path from source (node A) to destination (node F). However, since the route to node $\mathrm{F}$ is not yet learned, the RREQ is forwarded to node $\mathrm{F}$ and node $\mathrm{F}$ replies with an RREP. Each node maintains a list of source routes it has learned or overheard in its route cache. This enables the nodes to limit the utilization of the Route Discovery mechanism.

In Route Maintenance, node A is informed through a Route Error (RERR) packet that the route between node A and node $\mathrm{F}$ is broken. Thus, node $\mathrm{A}$ can either use another existing route from the route cache or begin the Route Discovery mechanism.

\section{B. Congestion Metric}

To obtain congestion information at each node, a method presented in [15] is used. In this method, two congestion metrics are used. The node's MAC layer utilization and the instantaneous transmission queue length. MAC layer utilization indicates the node's usage of the medium in the surrounding area. The usage of the medium is established by monitoring the operation state of the node. In this study, two operation states are used for the node, the busy state and the idle state. The instantaneous MAC layer usage is considered 0 when the node is idle and 1 when the node is busy. The wireless medium usage in this study is averaged within a simulation run time of $10 \mathrm{~ms}$ to indicate its usage.

Sometimes congestion occurs due to queue length packet backlog, and this may result in increased packet delays or dropped packets due to capacity limitations imposed on the queue length. Thus, instantaneous transmission queue length is used to indicate congestion triggered by queue length capacity.

\section{DSR Congestion Based Modification}

To enable the DSR protocol to use the congestion metrics discussed in the previous subsection, the route discovery mechanism of the protocol is modified as in [7],[15]. Upon reception of the RREQ packet, the router determines the packets intended destination. If it is the destination, it processes the packet and returns a RREP as in normal DSR operation. However, if RREQ is not directed to the node, it then checks the congestion metrics. If the congestion metrics indicates that there is a high level of congestion around the node, it silently discards the packet instead of forwarding it. This is because an additional flood of RREQ packets in a highly busy medium can only result in congestion aggravation. Thus, it is desirable for the discovered routes to bypass the congestive nodes to improve the performance of the network.

\section{Link Quality Metric}

Most existing WANETS network layer routing protocols commonly use minimum hop count (shortest route) as a routing metric. However, the minimum hop count metric presents several challenges for the wireless network [1],[6]. Firstly, it assumes that links are binary (meaning that they either exist or do not exist). Thus, a link that is not performing reasonably well for data is not good for broadcast packets. Secondly, shortest route increases hop range. Hence, the power received is degraded and packet loss is increased. Thirdly, several best shortest routes with equivalent distances, but dissimilar characteristics, may exist in a compact wireless network. Thus, a proper selection of the appropriate route is unlikely to be made. To alleviate inefficiencies provided by the minimum hop count metric, the Expected Transmission Count (ETX) metric was proposed in [6]. The ETX metric is a link quality metric that utilizes delivery ratios to overcome shortest route inefficiencies. It is calculated using the following expression.

$$
E T X=\frac{1}{d_{\text {forvard }} \mathrm{X} d_{\text {reverse }}},
$$

where $d_{\text {forward }}$ is the forward delivery ratio and $d_{\text {reverse }}$ is the reverse delivery ratio. The $d_{\text {forward }}$ is acquired by evaluating the probability of successful transmissions and the $d_{\text {reverse }}$ is acquired by evaluating the probability of acknowledged transmissions.

The two ratios $d_{\text {forward }}$ and $d_{\text {reverse }}$ are acquired using dedicated link probe packets of fixed size sent over a period $\tau$. The source ratio at any given instance $T$ is expressed as follows.

$$
r(t)=\frac{L p_{\text {Count }}(T-\lambda, T)}{\lambda / \tau}
$$

where $L p_{\text {Count }}(T-\lambda, T)$ is the count of fixed size packet probes received within the interval $\lambda$. The probe packet comprises of a count of probe packets received by the source from the surrounding nodes within the last $\lambda$ period. Thus, the receiver can calculate the $d_{\text {forward }}$ to the node which sent the probe.

\section{E. DSR Link Quality Based Modification}

To overcome the minimum hop count problems and enable the routing protocol to incorporate the ETX metric, the DSR Route Discovery mechanism is modified. RREP packets which emanate from RREQ's are alternatively stored in a link cache rather than a route cache that is utilized by the basic DSR. The route cache stores information about the entire route from source to destination, whilst, the link cache enables the separate storage of data about each connection in a source to destination path.

To deal with the link asymmetry problem, the nodes within the wireless network keep a list that contains nodes with one-way flows to unlisted nodes. If an RREP is unable to reach node $\mathrm{B}$ from node $\mathrm{A}$, node $\mathrm{B}$ is registered on the list 
with a status indicating one-way flow probability. Thus, node A will no longer send on RREQ's sent from node B. If one-way flow is not ascertained between node A and node B for a significant period, the status is changed to questionable one-way flow. Thus, if node B sends an RREQ to node A, node A will maintain the RREQ and reply to node $B$ with its own RREQ. If node B acknowledged receipt of the RREQ, then the original RREQ is sent onwards by node $A$, and node $B$ is erased from the list. However, if the RREQ from node A is not acknowledged, node A discards the original RREQ from node B. Nodes are erased from the list when two-way flow is established.

To enable DSR to incorporate the ETX metric, link loss ratios are measured as indicated in sub-section $\mathrm{C}$. In the simulations, IEEE $802.11 \mathrm{~b}$ probe packets comprising of a payload with 134 bytes is used. In addition, a probe packet is sent every millisecond and $\lambda$ is set at 10 milliseconds. If an RREQ originating from node $\mathrm{A}$ is routed onwards to node $\mathrm{C}$ by node $\mathrm{B}$, the address of node $\mathrm{B}$ and the link quality metric evaluated over the link between node $\mathrm{A}$ and $\mathrm{B}$ are attached to the RREQ forwarded to node $\mathrm{C}$, and the link cache is updated. This information will be added to the RREP which is sent back to node A. If the same request is initiated, the RREQ will be rerouted again if the link quality metric is better than the previous one. The Djikstra algorithm is used to select the best path that minimizes ETX from source to destination.

\section{Performance evaluation}

In this section, the performance of the cross-layer system is evaluated and validated through simulations. MATLAB 7.1 is used for rate adaptation evaluations and the $N S-2$ simulator version 2.29 is used for routing optimization evaluations.

\section{A. Simulation Methodology}

In order to effectively evaluate the performance of the cross-layer system, the rate adaptation simulation is conducted separately, and the results are used in NS-2 simulator to evaluate the performance of the routing protocol cross-layer optimizations. In the rate adaptation simulations, the nodes operate under the IEEE 802.11b DCF MAC layer scheme. For routing optimizations, an IEEE 802.11b-based NS-2 WMN network simulation model is created by randomly placing 4 nodes in a $500 \mathrm{~m} \times 500 \mathrm{~m}$ square area as shown in Figure 6. In the network model, node 0 is the video source, node 3 is the destination node and the rest of the nodes are intermediate nodes. The transmission range of each node is set to $100 \mathrm{~m}$. In this paper, a fixed WMN was considered. As a result, all the nodes were stationary during the simulations. A UDP agent was used to stream the video frame packets from the video source to the destination node. The maximum transmission packet size was set to 1024 bytes. A high quality video trace file called Verbose StarWarsIV available in [16] was used. The video trace file was MPEG4 encoded and contains 89998 frames. The frames comprise of 7500 I-frames, 22500 P-frames and 59998 B-frames. The frame rate was set to $30 \mathrm{fps}$.

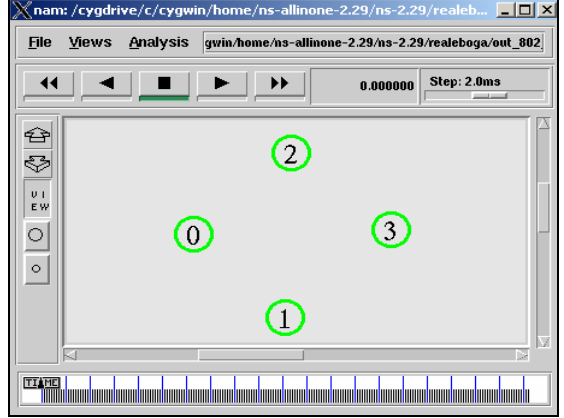

Figure 6: Network Simulation Model

\section{B. Simulation Results}

This subsection presents the cross-layer system simulation results based on the previously discussed simulation methodology.

\section{Rate adaptation}

For rate adaptation, the BER performance for different transmission rates is established as shown in Figure 7.The results achieved in Figure 7 were determined using Equations (2), (3) and (4) shown in section III and Equation (13)-(25) found in [14] which gives the bit error probability when transmitting an MPDU for each modulation scheme. The results shown in Figure 7 are used in throughput calculations to enable the threshold settings for the rate controller.

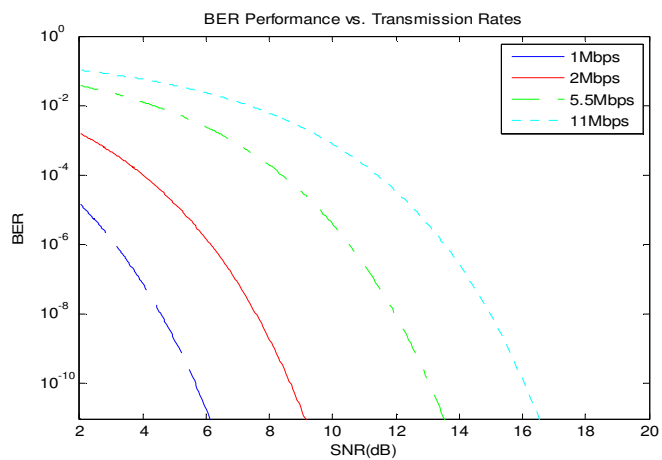

Figure 7: BER Performance for Different Transmission Rates

The throughput performance is established using the channel model and the throughput model discussed in section II $(\mathrm{C})$ as well as the results shown in Figure 7 . The values of the parameters as shown in Table 1 are used to determine the attainable throughput for each transmission rate.

In Figure 8, throughput performance for a fixed maximum MSDU length of 1500 bytes is shown. The attainable throughput for different transmission rates based on the channel quality conditions (RSS) is also shown in Figure 8. RSS was used instead of SNR as it was a more practical parameter that was available in NS-2 simulations. 
Table 1: IEEE 802.11b Simulation Parameters [20]

\begin{tabular}{ll}
\hline \hline \multicolumn{1}{c}{ Parameter } & \multicolumn{1}{c}{ Value } \\
\hline SLOT & $20 \mu \mathrm{s}$ \\
SIFS & $10 \mu \mathrm{s}$ \\
DIFS & $50 \mu \mathrm{s}$ \\
PLCP Preamble & $144 \mu \mathrm{s}$ \\
PLCP Header & $48 \mu \mathrm{s}$ \\
CWmin & 31 \\
CWmax & 1023 \\
MSDU Length & $1500 \mathrm{bytes}$ \\
Tx Power & $100 \mathrm{~mW}$ \\
Tx Distance & $500 \mathrm{~m}$ \\
Path Loss Exponent & 2.8 \\
& \\
\hline \hline
\end{tabular}

It is also shown in Figure 8 that the actual expected throughput was significantly reduced when compared with its respective transmission rate. This reduction in throughput is induced by the MAC and PHY layer overheads such as the MAC header, PLCP preamble and PLCP header.

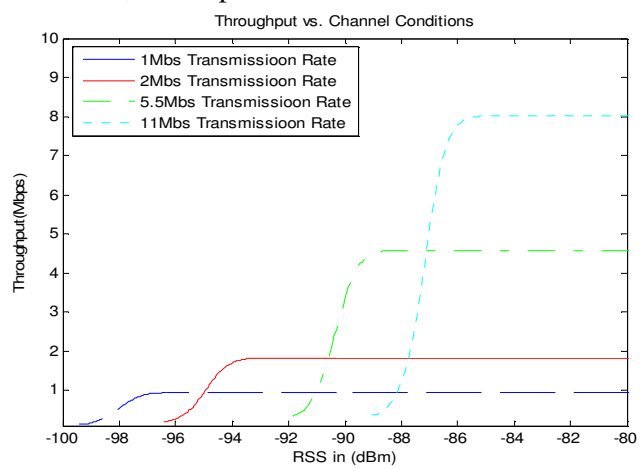

Figure 8: Throughput vs. RSS Curves for IEEE 802.11b

The rate controller thresholds extracted from Figure 8 are shown in Table 2. These thresholds are used in NS-2 simulations.

Table 2: Rate Controller Thresholds

\begin{tabular}{ll}
\hline \hline Rate (Mbps) & Rx Signal Strength $($ RSS $)$ \\
\hline 1 & $-95 \mathrm{dBm}>\mathrm{RSS}$ \\
2 & $-95 \mathrm{dBm}<\mathrm{RSS}<-90.5 \mathrm{dBm}$ \\
5.5 & $-90.5 \mathrm{dBm}<\mathrm{RSS}<-88.25 \mathrm{dBm}$ \\
11 & $-88.25 \mathrm{dBm}<\mathrm{RSS}$ \\
\hline \hline
\end{tabular}

\section{Routing Protocol}

For routing protocol optimizations, the DSR protocol discussed in sections IV was modified and implemented in $N S-2$ simulator. For congestion based modifications, the MAC layer utilization threshold was set at $30 \%$ and the interface queue length threshold was set at 50. These congestion metric thresholds values are used to initiate protocol optimization.

\section{Results and Discussions}

The performance comparison of a network not implementing the proposed cross-layer system and a network that incorporates the proposed cross-layer system was conducted to evaluate the performance of the proposed cross-layer system. The performance evaluation was carried out under high level congestion indicated by the congestion metrics thresholds values discussed in subsection 2 . Throughput, delay and jitter are used as network performance metrics.

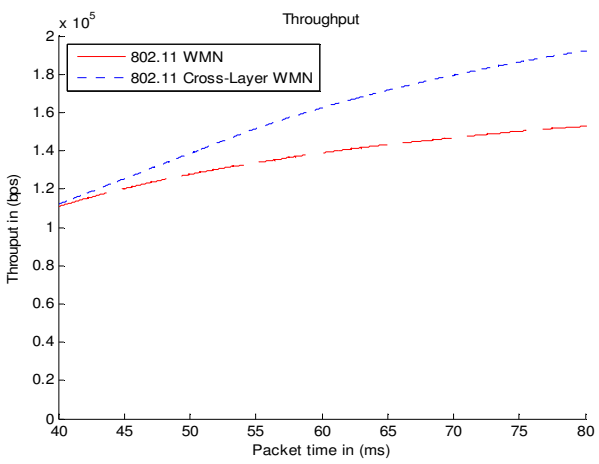

Figure 9: Throughput vs. Packet time

It is shown in Figure 9, 10 and 11 that the overall network performance was improved when cross-layer design is incorporated into a WMN. The graphs are plotted using the video source trace file and the destination node trace file generated by $N S-2$ simulator after a run time of $80 \mathrm{~ms}$.

In Figure 9, at 75 ms packet time, $150 \mathrm{Kbps}$ throughput for WMN without cross-layer optimization was achieved whilst $170 \mathrm{Kbps}$ throughput for cross-layer optimized network was achieved. Thus, a 13\% improvement was achieved by the cross-layer technique.
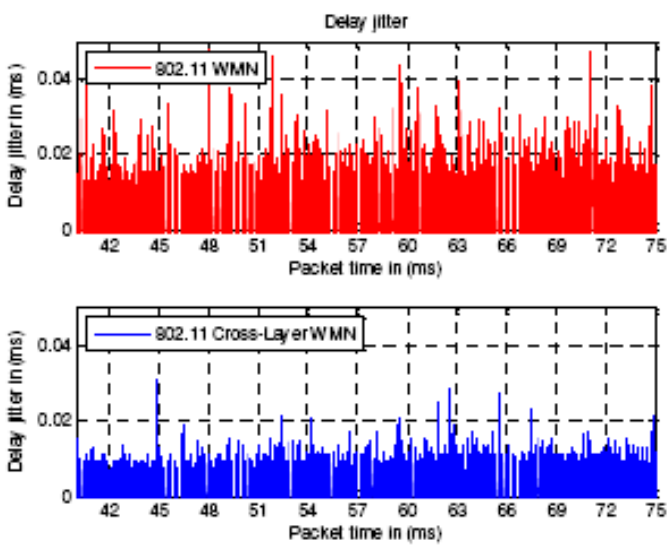

Figure 10: Delay Jitter vs. Packet time

In Figure 10, it is shown that the average jitter for the cross-layer optimized system is significantly lower when compared to the network without cross-layer optimization. For the network without cross-layer design, an average peak of $0.016 \mathrm{~ms}$ was achieved. This is significantly higher when compared to an average peak of $0.01 \mathrm{~ms}$ achieved in crosslayer optimized system.

Figure 11 illustrates the achieved average end-to-end delay. At $75 \mathrm{~ms}$ packet time, an average delay of $5 \mathrm{~ms}$ was achieved for the cross-layer optimized network whilst $21 \mathrm{~ms}$ was achieved for the network without cross-layer optimization. This results in a difference of $16 \mathrm{~ms}$ which is a 
considerable amount of delay in video streaming applications.

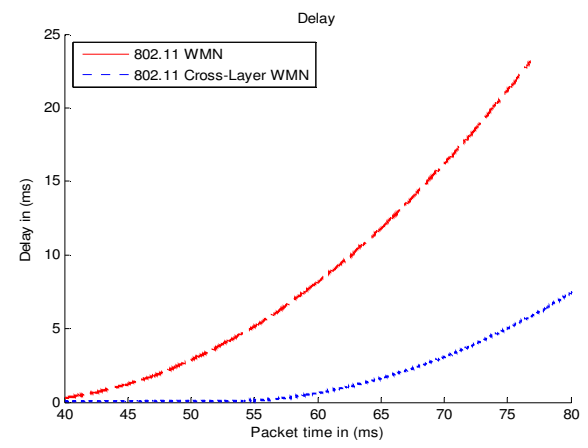

Figure 11: End-to-end Delay vs. Packet time

\section{Conclusion}

In this paper, rate adaptation, and congestion aware and link optimized routing are leveraged to implement a crosslayer technique that optimizes the UDP protocol for video streaming applications in wireless mesh networks. Congestion metrics and link quality metrics are integrated into the DSR protocol for optimal route selection in the network layer. Furthermore, rate adaptation is established in the data link layer for link reliability. Simulation results show that cross-layer techniques focused on the data link layer and network layer can be efficiently used to improve unreliable and congestion prone transport protocols such as UDP for delay sensitive and throughput constraint applications such as video streaming in wireless mesh networks. The results achieved indicate that better performance metrics need to be developed for routing protocols to be optimized for IEEE 802.11 multihop wireless mesh networks. They also show that multiple performance metrics needs to be integrated in routing protocols to facilitate the alleviation of video streaming challenges associated with WMNs.

\section{ACKNOWLEDGEMENTS}

The authors would like to thank the Telkom Centre of Excellence Program and F'SATIE at Tshwane University of Technology for making this research possible.

\section{REFERENCES}

[1] V.Chavoutier,D.Maniezo,C.E.Palazzi, M.Gerla, "Multimedia over Wireless Mesh Networks: Results from a Real Testbed Evaluation", The sixth Annual Ad hoc Networking workshop,Corfu,Greece,June 1215,2007 .

[2] Y.Andreopoulos,N.Mastronarde,M. van der Schaar, "Cross-Layer Optimized Video Streaming Over Wireless Multihop Mesh Networks", IEEE journal on Comunications, Vol.24,No.11,November 2006.

[3] Wei Wei, "Multipath Unicast and Multicast Video Communication Over Wireless Ad Hoc Networks", Ph.D Thesis, University of California, Fall 2004.

[4] L. Larzon, M. Degermark, and S. Pink, "Efficient Use of Wireless Bandwidth for Multimedia Applications", MoMuc '99, San Diego, November 1999, pp. 187-193.

[5] S. Bansal, R. Shorey, and A. Kherani, "Performance of TCP and UDP protocols in multi-hop multi-rate wireless networks", in: IEEE WCNC, Atlanta, USA, March 2004.

[6] D. S. J. De Couto, D. Aguayo, J. Bicket, and R. Morris, "A high throughput path metric for multi-hop wireless routing," in Proc. ACM Int. Conf. Mobile Comput. Netw, 2003, pp. 134-146.
[7] Ning Yang, "Congestion-Aware Cross-Layer Design for Wireless Ad Hoc Networks", Ph.D Thesis, University of South Florida, July 2004.

[8] Ivaylo Haratcherev, "Application-oriented Link Adaptation for IEEE 802.11", Ph.D Thesis, Delft University of Technology, March 2006, ISBN-10: 90-9020513-6, ISBN-13: 978-90-9020513-7

[9] http://www-cs students.stanford.edu/ dbfaria/publications.html

[10] L.N. Gumbi, D. Chatelain., M. Odhiambo, "Power-Efficient Algorithm for IEEE 802.11b Multi-Hop Networks" Proc. of Southern African Telecommunication Networks \& Applications Conference (SATNAC'06), Spier Wine Estate, South Western Cape, South Africa, September 2006

[11]T.S. Rappaport, Wireless Communications: Principles and Practice, Second Edition, (Upper Saddle River:Prentice Hall PTR, 2002).

[12] Javier del Prado Pavon, Sunghyun Choi, "Link Adaptation Strategy for IEEE 802.11 WLAN via Received Signal Strength Measurement", Proceedings of ICC'03, Vol. 2, pp. 1108-1113, Anchorage, Alaska, USA, May 2003

[13]Liljana Gravrilovska, Vladimir Atanasovski, "Influence of Packet Length on IEEE 802.11b Throughput Performance in Noisy Channels", Proceedings of $1^{\text {st }}$ International MAGNET Workshop D6.2.5, Shangai, China, 11-12 November 2004, ISBN 87-91696-36-4.

[14]D. B. Johnson, D. A. Maltz, and J. Broch. "The Dynamic Source Routing Protocol for Multihop Wireless Ad Hoc Networks," In Ad Hoc Networking, edited by Charles E. Perkins, chapter 5, pp. 139-172, Addison-Wesley, 2001.

[15] Y.-C. Hu and D. B. Johnson, "Exploiting Congestion Information in Network and Higher Layer Protocols in Multihop Wireless Ad Hoc Networks," In the 24th International conference on Distributed computing Systems (ICDCS 2004), pp.301-310, IEEE, Japan, March 2004

[16] VerboseStarwarsIV:http://www.tknbrlin.de/reseach/trace/pics/FrameTrace/mp4/index9c73.html/

[17]I. Haratcherev, K. Langendoen, R. Lagendijk and H. Sips, "Hybrid Rate Control for IEEE 802.11", ACM International Workshop on Mobility Management and Wireless Access Protocols (MobiWac), Philadelphia, PA, October 2004.

[18]A. Kamerman and L. Monteban. "WaveLAN-II: A High-performance wireless LAN for the unlicensed band", Bell Lab Technical Journal, pages 118-133, summer 1997.

[19]M. Lacage, H. Manshaei, and T. Turletti. "IEEE 82.11 Rate Adaptation: A Practical Approach", Rr-5208, Institut National De Recherche en Informatique et en Auomatique, 2004.

[20]B. Sadeghi, V. Kanodia, A. Sabharwal, and E. Knightly. "Opportunistic Media Access for Multirate Ad Hoc Networks", 2002.

[21]J. Taal, H. Sips, I. Langedijk, K. Langendoen, and I. Haratcherev. "Automatic IEEE 802.11 Rate Control for Streaming Applications", In Wireless Communications and mobile Computing, Netherlands, pp. 421-437, 2005.

[22] J. Broch, D.A. Maltz, D.B. Johnson, Y.-C. Hu, and J. Jetcheva. "A Performance Comparison of Multi-Hop Wireless Ad Hoc Network Routing Protocols", In the fourth annual ACM/IEEE International Conference on Mobile Computing and Networking (MobiCom'98), pages 85-97, October 1998

[23]D.B. Johnson, D.A. Maltz, and J. Broch. "The Dynamic Source Routing Protocol for Multihop Wireless Ad Hoc Networks", In Ad Hoc Networking, Edited by Charles E. Perkins, Chapter 5, pp. 139-172, Addison-Wesley, 2001.

N.H. Moleme obtained her B.Tech Degree from Tshwane University of Technology in 2006. She is currently working towards a double degree, (M.Sc with F'SATIE, and M.Tech with Tshwane University of Technology). Her research interests include rate adaptation algorithms, network protocols, and multimedia applications in wireless networks. 